\title{
Marcinkiewicz-Zygmund-Type Inequalities Trigonometric Interpolation on Non-Uniform Grids and Unconditional Schauder Bases in Besov Spaces on the Torus
}

\author{
K. V. Runovski and W. Sickel
}

\begin{abstract}
We investigate extensions of the classical Marcinkiewicz-Zygmund inequality to non-uniform grids. Then we show how this inequality can be used to characterize Besov spaces on the torus by means of approximation by corresponding interpolatory polynomials. One of these characterizations can be applied to the construction of unconditional Schauder bases.

Keywords: Marcinkiewicz-Zygmund inequalities, trigonometric interpolation on non-uniform grids, rate of convergence in Besov spaces on the torus, unconditional Schauder bases in Besov spaces on the torus
\end{abstract}

AMS subject classification: 41 A 25, 41 A 27, 42 A 05, 42 A 15, 46 E 35

\section{Introduction}

The goal of the paper consists in an extension of the Marcinkiewicz-Zygmund inequality to the case of non-uniform distributed knots. Based on this generalization we investigate the rate of convergence of corresponding trigonometric interpolation operators. In a certain sense these results may be understood as some sort of robustness of approximation by interpolatory trigonometric polynomials. Using an appropriate characterization of the periodic Besov spaces $B_{p q}^{s}$ we construct unconditional bases for $B_{p q}^{s}$ based on these interpolation operators. These bases do not fit in the scheme of wavelet bases; however, they are in the wavelet-spirit.

The paper is organized as follows. In Section 2 we deal with Marcinkiewicz-Zygmund type inequalities with respect to non-uniform grids. In the next Section 3 we describe consequences for approximation properties of corresponding sequences of interpolatory trigonometric polynomials. Section 4 is devoted to the construction of unconditional bases in Besov spaces. Here we use the fundamental functions of interpolation with respect to certain non-uniform grids. Finally, in Section 5 we investigate some examples.

K. V. Runovski: Friedrich-Schiller-Úniversität, Math. Inst., D - 07743 Jena

W. Sickel: Friedrich-Schiller-Universität, Math. Inst., D - 07743 Jena

ISSN 0232-2064 / \$2.50 (c) Heldermann Verlag Berlin 


\section{Marcinkiewicz-Zygmund type inequalities for non-uniform grids}

Classically one deals with the set of equally distributed knots

$$
x_{n k}=\frac{2 \pi k}{2 n+1} \quad(k=0,1, \ldots, 2 n) .
$$

Then the Marcinkiewicz-Zygmund inequality may be stated as follows. Let $1<p<\infty$. Then there exist constants $A_{p}, B_{p}>0$ such that

$$
B_{p}\left(\frac{1}{2 n+1} \sum_{k=0}^{2 n}\left|T_{n}\left(x_{n k}\right)\right|^{p}\right)^{\frac{1}{p}} \leq\left(\int_{0}^{2 \pi}\left|T_{n}(x)\right|^{p} d x\right)^{\frac{1}{p}} \leq A_{p}\left(\frac{1}{2 n+1} \sum_{k=0}^{2 n}\left|T_{n}\left(x_{n k}\right)\right|^{p}\right)^{\frac{1}{p}}
$$

holds for all trigonometric polynomials $T_{n}$ of degree at most $n$ and all non-negative integers $n$. Here we are asking for conditions on a matrix $Y=\left\{y_{n k}\right\}_{\substack{0 \leq 0 \\ 0 \leq k \leq 2 n}}$, where $0 \leq y_{n 0}<y_{n 1}<\ldots<y_{n 2 n}<2 \pi$ holds for all $n$ (but the knots $y_{n k}$ need not be equally distributed), such that corresponding inequalities are valid without supposing (1).

We start with some notations. For brevity we shall make use of

$$
\left\|f \mid Y_{n}, p\right\|=\left(\frac{1}{2 n+1} \sum_{k=0}^{2 n}\left|f\left(y_{n k}\right)\right|^{p}\right)^{\frac{1}{p}} \quad \text { if } \quad Y_{n}=\left\{y_{n k}\right\}_{k=0}^{2 n}
$$

As usual, $L_{p}$ denotes the set of all complex-valued Lebesgue-measurable functions which are $p$-th power integrable, equipped with the norm

$$
\left\|f \mid L_{p}\right\|=\left(\int_{0}^{2 \pi}|f(x)|^{p} d x\right)^{\frac{1}{p}} \quad(1 \leq p<\infty)
$$

By $C$ we mean the set of $2 \pi$-periodic continuous functions. Further, $\mathbb{N}$ denotes the set of natural numbers, $\mathbb{N}_{0}$ the set of natural numbers including zero, $\mathbb{R}$ is used for the real line, and $\mathbb{C}$ for the complex plane. The symbol $[x]$ is used to denote the integer part of a real number $x \in \mathbb{R}$.

There are various ways to attack an extension of (2). First we deal with the left part of (2); that means we are asking for the existence of some constant $C_{p}$ such that

$$
\left(\frac{1}{2 n+1} \sum_{k=0}^{2 n}\left|T_{n}\left(y_{n k}\right)\right|^{p}\right)^{\frac{1}{p}} \leq C_{p}\left(\int_{0}^{2 \pi}\left|T_{n}(x)\right|^{p} d x\right)^{\frac{1}{p}}
$$

holds for all trigonometric polynomials of degree at most $n$ and under certain conditions on the matrix $Y$. 
Theorem 1. Let $0<p<\infty$. Let $\mu$ be a non-decreasing function on $\mathbb{R}$ satisfying $\mu(x+2 \pi)-\mu(x) \equiv$ const. We put

$$
\omega_{\mu}(\delta)=\sup _{0<y-x \leq \delta}(\mu(y)-\mu(x)) \quad(\delta>0)
$$

Then the estimate

$$
\left(\int_{0}^{2 \pi}\left|T_{n}(x)\right|^{p} d \mu(x)\right)^{\frac{1}{p}} \leq C_{p}\left(\frac{n}{2 \pi} \omega_{\mu}\left(\frac{1}{n}\right)\right)^{\frac{1}{p}}\left(\int_{0}^{2 \pi}\left|T_{n}(x)\right|^{p} d x\right)^{\frac{1}{p}}
$$

holds true for all trigonometric polynomials $T_{n}$ of degree at most $n$. Here $C_{p}$ is the constant which appears in the inequality

$$
\left(\frac{2 \pi}{n} \sum_{k=0}^{n-1} \max _{x \in I_{k}}\left|T_{n}(x)\right|^{p}\right)^{\frac{1}{p}} \leq C_{p}\left\|T_{n} \mid L_{p}\right\|
$$

where again $T_{n}$ varies over the set of all trigonometric polynomials $T_{n}$ of degree at most $n$ and $I_{k}=\left[\frac{2 \pi k}{n}, \frac{2 \pi(k+1)}{n}\right]$.

Proof. We have

$$
\begin{aligned}
\int_{0}^{2 \pi}\left|T_{n}(x)\right|^{p} d \mu(x) & =\sum_{k=0}^{n-1} \int_{I_{k}}\left|T_{n}(x)\right|^{p} d \mu(x) \\
& \leq \sum_{k=0}^{n-1} \max _{x \in I_{k}}\left|T_{n}(x)\right|^{p} \int_{I_{k}} d \mu(x) \\
& \leq\left(\frac{n}{2 \pi} \omega_{\mu}\left(\frac{1}{n}\right)\right) \sum_{k=0}^{n-1} \frac{2 \pi}{n} \max _{x \in I_{k}}\left|T_{n}(x)\right|^{p}
\end{aligned}
$$

The estimate is finished by applying (6), which may be found in [16: Theorem 3.3.5]

Remark 1. We do not have exact values of the constant $C_{p}$, even we do not know, whether it depends on $p$ (the proof of (6) in [16: Subsection 3.3.5] makes use of some maximal inequalities, which is probably not an appropriate way for selecting a "good" constant). Based on a particular identity for trigonometric polynomials (cf. [14]), we only have a bound for $C_{p}$ : it holds

$$
C_{p} \leq\left(\frac{2}{\pi}\right)^{\frac{1}{p}}\left(\frac{17}{4}+\log 3\right) .
$$

We are not interested in situations where more than two knots are coming close to each other. So we require an additional regularity condition to the admissible matrices. We always assume that the matrix $Y$ is a weakly disturbed version of the matrix $X=$ $\left\{x_{n k}\right\}$ (cf. (1)). 


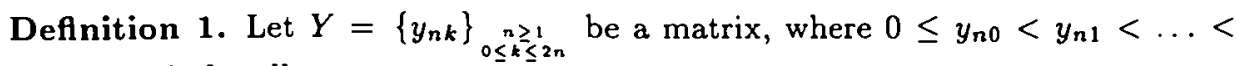
$y_{n 2 n}<2 \pi$ holds for all $n$.

(i) We call $Y$ almost regular if

$$
\frac{2 \pi\left(k-\frac{1}{2}\right)}{2 n+1} \leq y_{n k}<\frac{2 \pi\left(k+\frac{1}{2}\right)}{2 n+1}
$$

holds for all $k$ and all $n$.

(ii) Let $0 \leq \sigma \leq \frac{1}{2}$. We call $Y$ almost regular of order $\sigma$ if

$$
\frac{2 \pi(k-\sigma)}{2 n+1} \leq y_{n k}<\frac{2 \pi(k+\sigma)}{2 n+1}
$$

holds for all $k$ and all $n$.

Remark 2. If $Y$ is almost regular, then

$$
\left\|\left.f\left|Y_{n}, p \|^{p}=\frac{1}{2 n+1} \sum_{k=0}^{2 n}\right| f\left(y_{n k}\right)\right|^{p}=\int_{0}^{2 \pi}|f(x)|^{p} d \mu_{n}(x)\right.
$$

and

$$
n \omega_{\mu_{n}}\left(\frac{1}{n}\right) \leq \frac{2 n}{2 n+1} \leq 1
$$

Hence, for an almost regular matrix $Y$ inequality (5) is true with the constant $C_{p}$ appearing in (6).

Theorem 2. Let $1<p<\infty$. Let

$$
\sigma_{p}=\frac{1}{\pi A_{p} C_{p}}
$$

Here $A_{p}$ and $C_{p}$ are the constants taken from (2) and (6), respectively. Let $Y$ be an almost regular matrix of order $\sigma<\sigma_{p}$. Then

$$
\left\|T_{n}\left|L_{p}\left\|\leq \frac{1}{\pi C_{p}\left(\sigma_{p}-\sigma\right)}\right\| T_{n}\right| Y_{n}, p\right\|=D_{p}\left\|T_{n} \mid Y_{n}, p\right\|
$$

holds for all trigonometric polynomials of degree at most $n$.

Proof. We shall apply (2). The set of equally distributed knots defined in (1) we denote by $X_{n}$. It follows that

$$
\begin{aligned}
\left\|T_{n} \mid L_{p}\right\| & \leq A_{p}\left\|T_{n} \mid X_{n}, p\right\| \\
& \leq A_{p}\left\|T_{n} \mid Y_{n}, p\right\|+A_{p}\left\{\frac{1}{2 n+1} \sum_{k=0}^{2 n}\left|T_{n}\left(x_{n k}\right)-T_{n}\left(y_{n k}\right)\right|^{p}\right\}^{\frac{1}{p}} \\
& \leq A_{p}\left\|T_{n} \mid Y_{n}, p\right\|+\frac{2 \pi \sigma A_{p}}{2 n+1}\left\{\frac{1}{2 n+1} \sum_{k=0}^{2 n}\left|T_{n}{ }^{\prime}\left(\xi_{k}\right)\right|^{p}\right\}^{\frac{1}{p}}
\end{aligned}
$$


for suitable chosen $\Xi=\left\{\xi_{n k}\right\}_{n, k}$ and $\Xi$ is also almost regular of order $\sigma$. Applying first Theorem 1 and afterwards Stechkin's inequality, given by $\left\|T_{n}^{\prime}\right\|_{p} \leq n\left\|T_{n}\right\|_{p}$ (cf. [19]), we obtain

$$
\left\|T_{n}\left|L_{p}\left\|\leq A_{p}\right\| T_{n}\right| Y_{n}, p\right\|+\frac{2 \pi \sigma A_{p} C_{p} n}{2 n+1}\left\|T_{n} \mid L_{p}\right\|
$$

By assumption $\pi \sigma A_{p} C_{p}<1$. Hence

$$
\left\|T_{n}\left|L_{p}\left\|\leq \frac{A_{p}}{1-\pi \sigma A_{p} C_{p}}\right\| T_{n}\right| Y_{n}, p\right\|
$$

which completes the proof

Remark 3. We continue with a comment to the constants $A_{p}$, and $\sigma_{p}$. In particular, in case $p=2$ it is known that

$$
A_{2}=\sqrt{2 \pi}
$$

(cf. [22: Volume II/p. 8]). Using (7) this gives $\sigma_{2}>\frac{1}{34}$. Information about the asymptotic behaviour of $A_{p}$ for $p$ tending to 1 or $\infty$ is available in [11].

Remark 4. There are simple examples to show that $\sigma<\frac{1}{2}$ is necessary in Theorem 2. We choose

$$
T_{n}(x)=\sin \left(n x-\frac{n \pi}{2 n+1}\right) \quad(n \in \mathbb{N})
$$

and

$$
y_{n 0}=0, \quad y_{n k}=\frac{\pi}{2 n+1}+\frac{(k-1) \pi}{n} \quad(k=1,2, \ldots, 2 n) .
$$

Then $Y$ is almost regular with $\sigma=\frac{1}{2}$,

$$
\left\|T_{n}\left|Y_{n}, p \|=(2 n+1)^{-\frac{1}{p}}\right| \sin \left(\frac{n \pi}{2 n+1}\right) \mid,\right.
$$

and $\left\|T_{n}\left|L_{p}\|=\| \sin y\right| L_{p}\right\|$.

\section{On the rate of convergence of trigonometric interpolation and periodic Besov spaces}

Our aim is to study the rate of convergence of interpolatory polynomials in dependence of the regularity of the approximated function. It turns out that the correct classes to do this are the periodic Besov spaces. Therefore we start with a short description of their properties.

3.1 Besov spaces of periodic functions. We follow [16: Chapter 3]. Let $D_{\pi}$ and $D_{\pi}{ }^{\prime}$ denote the set of all complex-valued, $2 \pi$-periodic, and infinitely differentiable functions and its dual space, respectively. Furthermore, we put

$$
\hat{f}(k)=f^{\wedge}(k)=(2 \pi)^{-1} f\left(e^{i k x}\right) \quad\left(k \in \mathbb{Z}, f \in D_{\pi}^{\prime}\right) .
$$


Then any $f \in D_{\pi}{ }^{\prime}$ can be represented by its Fourier series

$$
f=\sum_{k \in \mathbf{Z}} \hat{f}(\dot{k}) e^{i k x} \quad \text { (convergence in } D_{\pi}{ }^{\prime} \text { ). }
$$

To introduce Besov spaces we need a smooth decomposition of unity. Therefore, let $\psi$ be an infinitely differentiable function satisfying

$$
\psi(x)= \begin{cases}1 & \text { if }|x| \leq 1 \\ 0 & \text { if }|x| \geq \frac{3}{2}\end{cases}
$$

Next we put

Hence, we have

$$
\left.\begin{array}{l}
\varphi_{0}(x)=\psi(x) \\
\varphi_{1}(x)=\psi\left(2^{-1} x\right)-\psi(x) \\
\varphi_{\ell}(x)=\varphi_{1}\left(2^{-\ell+1} x\right) \quad(\ell \geq 2)
\end{array}\right\}
$$

$$
\sum_{l=0}^{\infty} \varphi_{\ell}(x)=1 \quad \text { for all } x \in \mathbb{R}
$$

Definition 2. Let $1 \leq p \leq \infty, 0<q \leq \infty$ and $s \in \mathbb{R}$. Then we put

$$
B_{p q}^{s}=\left\{f \in D_{\pi}^{\prime}:\left\|f \mid B_{p q}^{s}\right\|=\left(\sum_{\ell=0}^{\infty} 2^{\ell s q}\left\|\sum_{k \in \mathbf{Z}} \varphi_{\ell}(k) \hat{f}(k) e^{i k x} \mid L_{p}\right\|^{q}\right)^{\frac{1}{q}}<\infty\right\}
$$

$(q<\infty)$ and

$$
B_{p \infty}^{s}=\left\{f \in D_{\pi}^{\prime}:\left\|f\left|B_{p \infty}^{s}\left\|=\sup _{\ell \geq 0} 2^{\ell s}\right\| \sum_{k \in \mathbf{Z}} \varphi_{\ell}(k) \hat{f}(k) e^{i k x}\right| L_{p}\right\|<\infty\right\} .
$$

Remark 5. All spaces above are quasi-Banach spaces (Banach spaces if $q \geq 1$ ). They are independent of the special choice of $\psi$ (equivalent quasi-norms). These spaces of periodic functions are extensively investigated in [6: Chapter 5] and in [16: Chapter 3].

Remark 6. For $s>0$ the above somewhat complicated definition of $B_{p q}^{s}$ coincides with those one given by means of moduli of smoothness or derivatives and differences etc. (cf., e.g., [16: Subsection 3.5.4]).

Remark 7. Of some interest will be the following:

$$
B_{p q}^{s} \hookrightarrow C \quad \text { if and only if } \quad\left\{\begin{array}{l}
\text { either } s>\frac{1}{p} \\
\text { or } s=\frac{1}{p} \text { and } 0<q \leq 1
\end{array},\right.
$$

(cf. [6: Sections 6.2, 6.3] or [16: Subsection 3.5.5]).

Let us recall the well-known characterization of $B_{p q}^{s}$ based on best approximation: as usual we put

$$
E_{n}\left(f, L_{p}\right)=\inf \left\|f-g \mid L_{p}\right\|
$$

where the infinum is taken over all trigonometric polynomials of degree at most $n$. 
Proposition 1 (see [6, Section 5.6] or [16: Subsection 3.7.1]). Let $0<q \leq \infty, 1 \leq$ $p \leq \infty$ and $s>0$. Then $f \in B_{p q}^{s}$ if and only if

$$
(n+1)^{9-\frac{1}{q}} E_{n}\left(f, L_{p}\right) \in \ell_{q} .
$$

Moreover,

$$
\left\|f \mid L_{p}\right\|+\left(\sum_{n=0}^{\infty}\left((1+n)^{s-\frac{1}{q}} E_{n}\left(f, L_{p}\right)\right)^{q}\right)^{\frac{1}{q}}
$$

yields an equivalent quasi-norm on $B_{p q}^{s}$.

As it is also well-known one can replace $E_{n}\left(f, L_{p}\right)$ by the error of some concrete approximation processes, e.g. de la Vallée-Poussin means. For us it will be sufficient to consider the following modified version of those means. Let $\psi$ be the function defined in. (13). Then we put

$$
\left(\psi_{n} f\right)(x)=\sum_{k \in \mathbf{Z}} \psi\left(\frac{k}{n}\right) \hat{f}(k) e^{i k x} \quad\left(n \in \mathbb{N}, f \in D_{\pi}^{\prime}\right) .
$$

Proposition 2 (see [6: Section 5.2] or [16: Subsection 3.7.4]). Let $0<q \leq \infty, 1 \leq$ $p \leq \infty$, and $s>0$. Then $f \in B_{p q}^{s}$ if and only if

$$
(n+1)^{s-\frac{1}{q}}\left\|f-\psi_{n} f \mid L_{p}\right\| \in \ell_{q} .
$$

Moreover,

$$
\left\|f \mid L_{p}\right\|+\left(\sum_{n=0}^{\infty}\left((n+1)^{s-\frac{1}{q}}\left\|f-\psi_{n} f \mid L_{p}\right\|\right)^{q}\right)^{\frac{1}{q}}
$$

yields an equivalent quasi-norm on $B_{p q}^{s}$.

3.2 Approximation of functions by interpolatory polynomials. In this part we consider matrices $Y=\left\{y_{n, k}\right\}_{n, k}$ which are almost regular of order $\sigma<\sigma_{p}$. Thanks to the periodicity of the underlying function spaces we may consider also the following more general classes.

Definition 3. We say a matrix $Y=\left\{y_{n, k}\right\}_{n, k}$ satisfies the regularity condition $\left(\mathcal{R}_{p, \sigma}\right)$ if there exists a sequence $\left\{z_{n}\right\}_{n=0}^{\infty}$ of real numbers such that the matrix $\widetilde{Y}$ defined by

$$
\tilde{y}_{n k}=y_{n k}-z_{n} \quad\left(k=0, \ldots, 2 n ; n \in \mathbb{N}_{0}\right)
$$

is almost regular of order $\sigma<\sigma_{p}$ (cf. (9)).

Of course, if the matrix satisfies the regularity condition $\left(\mathcal{R}_{p, \sigma}\right)$, then we have the equivalence of the discrete norm $\left\|T_{n} \mid Y_{n}, p\right\|$ and the continuous norm $\left\|T_{n} \mid L_{p}\right\|$ on each level $n$ and the corresponding constants do not depend on $n$ (as before, if $Y$ is a given matrix, then $Y_{n}$ denotes its $n$-th row). 
To such a given matrix $Y$ and to any periodic function $f \in C$ we associate a sequence of interpolatory polynomials $I_{n}$ by

$$
\left(I_{n} f\right)(x)=\sum_{k=0}^{2 n} f\left(y_{n k}\right) \ell_{n k}(x)
$$

where the fundamental functions $\ell_{n k}$ are defined to be

$$
\ell_{n k}(x)=\prod_{j \in\{0,1, \ldots, 2 n\} \backslash\{k\}} \frac{\sin \left(\frac{x-y_{n j}}{2}\right)}{\sin \left(\frac{y_{n k}-y_{n j}}{2}\right)} \quad(x \in \mathbb{R}, n \in \mathbb{N}, k=0, \ldots, 2 n)
$$

and $\ell_{00}(x)=1$.

Later on we need that the polynomials $I_{n}$ are projections onto the set of trigonometric polynomials of order at most $n$, that means

$$
I_{n} T_{n}=T_{n}
$$

for all trigonometric polynomials $T_{n}$ of degree at most $n$ (cf. [22: Volume 2, Chapter 10/p.1]).

Theorem 3. Let $1<p<\infty$. If the matrix $Y$ satisfies the regularity condition $\left(\mathcal{R}_{p, \sigma}\right)$, then there exists a constant $c_{p, \sigma}$ such that

$$
\left\|f-I_{n} f\left|L_{p}\left\|\leq c_{p, \sigma}(1+n)^{-\frac{1}{p}}\right\| f\right| B_{p 1}^{\frac{1}{p}}\right\|
$$

holds for all $f \in B_{p 1}^{\frac{1}{p}}$ and all $n \in \mathbb{N}_{0}$.

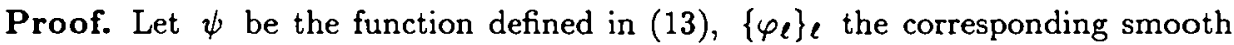
decomposition of unity from (14), and $\psi_{n} f$ the de la Vallée-Poussin means introduced in (15). Let $\left[\frac{n}{2}\right]$ be the integer part of $\frac{n}{2}$. Then we have

$$
f-I_{n} f=f-\psi_{\left[\frac{n}{2}\right]} f+I_{n}\left(\psi_{\left[\frac{n}{2}\right]} f-f\right) .
$$

For convenience we introduce the abbreviation

$$
f_{\ell}(x)=\sum_{k \in \mathbf{Z}} \varphi_{\ell}(k) \hat{f}(k) e^{i k x} \quad\left(x \in \mathbb{R}, \ell \in \mathbb{N}_{0}\right) .
$$

Then, if $2^{r} \leq n<2^{r+1}$, the identity

$$
\begin{aligned}
\left(\psi_{\left[\frac{n}{2}\right]} f-f\right)(x) & =\sum_{\ell=r-1}^{\infty}\left(\sum_{k \in \mathbf{Z}} \varphi_{\ell}(k)\left(\psi_{\left[\frac{n}{2}\right]} f-f\right)^{\wedge}(k) e^{i k x}\right) \\
& =\sum_{\ell=0}^{\infty}\left(\psi_{\left[\frac{n}{2}\right]} f-f\right)_{\ell+r-1}(x)
\end{aligned}
$$


holds. In this situation Theorem 2 yields

$$
\begin{aligned}
\left\|I_{n}\left(f-\psi_{\left[\frac{n}{2}\right]} f\right) \mid L_{p}\right\| & \leq \sum_{\ell=0}^{\infty}\left\|I_{n}\left(f-\psi_{\left[\frac{n}{2}\right]} f\right)_{\ell+r-1} \mid L_{p}\right\| \\
& \leq D_{p} \sum_{\ell=0}^{\infty}\left\|I_{n}\left(f-\psi_{\left[\frac{n}{2}\right]} f\right)_{\ell+r-1} \mid Y_{n}, p\right\| \\
& =D_{p} \sum_{\ell=0}^{\infty}\left\|\left(f-\psi_{\left[\frac{n}{2}\right]} f\right)_{\ell+r-1} \mid Y_{n}, p\right\| .
\end{aligned}
$$

Next we make use of a simple observation. Any row $Y_{n}$ of a matrix $Y$ satisfying the regularity condition $\left(\mathcal{R}_{p, \sigma}\right)$ can be complemented to a matrix $Z$ such that $Y_{n}=Z_{n}$ and $Z_{n} \subset Z_{n+\ell}(\ell \geq 1)$. Since we are going to apply Theorem 1 only minimal regularity properties of the corresponding sequences $\left\{z_{n k}\right\}$ are needed. In fact, we use

$$
\frac{2 \pi\left(k-\frac{1}{2}\right)}{2\left(2^{r+m}+2^{\ell}\right)+1} \leq z_{\left(2^{r+m}+2^{\ell}\right) k} \leq \frac{2 \pi\left(k+\frac{1}{2}\right)}{2\left(2^{r+m}+2^{\ell}\right)+1}
$$

for $k=0, \ldots, 2\left(2^{r+m}+2^{\ell}\right)$ and $\ell \geq 1$ where $m \in \mathbb{N}$ has to be chosen sufficiently large but independent of $n$. Applying Theorem 1 with respect to $Z_{2^{r+m}+2^{\ell}}$ we obtain

$$
\begin{aligned}
\left\|\left(f-\psi_{\left[\frac{n}{2}\right]} f\right)_{\ell+r-1} \mid Y_{n}, p\right\| & \leq\left\|\left(f-\psi_{\left[\frac{n}{2}\right]} f\right)_{\ell+r-1} \mid Z_{2^{r+m}+2^{\ell}, p}\right\| \\
& \leq C_{p}\left(\frac{2^{\ell+r+m}+1}{2 n+1}\right)^{\frac{1}{p}}\left\|\left(f-\psi_{\left[\frac{n}{2}\right]} f\right)_{\ell+r-1} \mid L_{p}\right\|
\end{aligned}
$$

and finally

$$
\begin{aligned}
\left\|I_{n}\left(f-\psi_{\left[\frac{n}{2}\right]} f\right) \mid L_{p}\right\| & \leq D_{p} C_{p} \sum_{\ell=0}^{\infty}\left(\frac{2^{\ell+r+m}+1}{2 n+1}\right)^{\frac{1}{p}}\left\|\left(f-\psi_{\left[\frac{n}{2}\right]} f\right)_{\ell+r-1} \mid L_{p}\right\| \\
& \leq C \sum_{\ell=0}^{\infty} 2^{\frac{\ell}{p}}\left(\left\|f_{\ell+r-1}\left|L_{p}\|+\|\left(\psi_{\left[\frac{n}{2}\right]} f\right)_{\ell+r-1}\right| L_{p}\right\|\right)
\end{aligned}
$$

where $C$ does not depend on $Y, Z, f$ and $n$. By a Fourier multiplier assertion we know

$$
\sup _{n \in \mathbb{N}_{0}}\left\|\psi_{n} g\left|L_{p}\|\leq c\| g\right| L_{p}\right\|
$$

for all $g \in L_{p}$ (cf. e.g. [16: Theorem 3.3.4]). Taking this into account (18), (20) and Proposition 2 are leading to

$$
\begin{aligned}
\left\|f-I_{n} f \mid L_{p}\right\| & \leq c\left((1+n)^{-\frac{1}{p}}\left\|f\left|B_{p 1}^{\frac{1}{p}}\left\|+2^{-\frac{r}{p}} \sum_{\ell=0}^{\infty} 2^{\frac{\ell+r-1}{p}}\right\| f_{\ell+r-1}\right| L_{p}\right\|\right) \\
& \leq c^{\prime}(1+n)^{-\frac{1}{p}}\left\|f \mid B_{p 1}^{\frac{1}{p}}\right\|
\end{aligned}
$$

for some constant $c^{\prime}$ independent of $n$ and $f$ 
Remark 8. An inspection of the proof of Theorem 3 shows that we have even proved more: for $f \in B_{p 1}^{\frac{1}{p}}$ it holds

$$
n^{\frac{1}{p}}\left\|f-I_{n} f \mid L_{p}\right\| \longrightarrow 0 \quad \text { as } n \rightarrow \infty
$$

For this one has to use

$$
n^{\frac{1}{p}}\left\|f-\psi_{\left[\frac{n}{2}\right]} f \mid L_{p}\right\| \longrightarrow 0 \quad \text { as } n \rightarrow \infty
$$

and

$$
\sum_{\ell=0}^{\infty} 2^{\frac{\ell+r}{p}}\left\|f_{\ell+r} \mid L_{p}\right\| \longrightarrow 0 \quad \text { as } r \rightarrow \infty \text {. }
$$

Furthermore, there is no hope to replace $B_{p 1}^{\frac{1}{p}}$ by $B_{p q}^{\frac{1}{p}}$ with $q>1$ in (17) and (22). That follows immediatly from Remark 7.

Remark 9. Similar results but restricted to the matrix $X=\left\{x_{n k}\right\}_{n, k}$ (cf. (1)) are derived in Oskolkov [8], Prestin [12] and Sickel [17].

Shortly we discuss an extension to $p=\infty$.

Lemma 1. Let $1 \leq p<\infty$. If $Y$ is a matrix satisfying the regularity condition $\left(\mathcal{R}_{p, \sigma}\right)$, then

$$
\left\|f-I_{n} f \mid C\right\| \longrightarrow 0 \quad \text { as } n \rightarrow \infty
$$

holds for all $f \in B_{p 1}^{\frac{1}{p}}$.

Proof. From Proposition 2 we know

$$
\left\|f-\psi_{\left[\frac{n}{2}\right]} f \mid C\right\| \longrightarrow 0 \quad \text { as } n \rightarrow \infty
$$

for any $f \in B_{\infty q}^{0}$, with $q<\infty$. Because of $B_{p, 1}^{\frac{1}{p}} \hookrightarrow B_{\infty 1}^{0}$ this holds for those $f$ we are interested in. Next we apply Nikol'skij's inequality (cf. [6: Subsection 3.3.5] or [16: Subsection 3.3.2]). It follows

$$
\left\|I_{n}\left(f-\psi_{\left[\frac{n}{2}\right]} f\right)\left|C\left\|\leq c(1+n)^{\frac{1}{p}}\right\| I_{n}\left(f-\psi_{\left[\frac{n}{2}\right]} f\right)\right| L_{p}\right\| .
$$

Remark 8 complements the proof

3.3 Characterization of periodic Besov spaces. Next we formulate and prove a counterpart to Proposition 2.

Theorem 4. Let $1<p<\infty, 0<q \leq \infty$, and $s>\frac{1}{p}$. Let $Y$ be a matrix satisfying the regularity condition $\left(\mathcal{R}_{p, \sigma}\right)$. Then the following assertions are equivalent:

(i) $f \in B_{p q}^{s}$. 
(ii) $f \in C$ and

$$
\left|f\left(y_{00}\right)\right|+\left(\sum_{n=0}^{\infty}\left((1+n)^{s-\frac{1}{q}}\left\|f-I_{n} f \mid L_{p}\right\|\right)^{q}\right)^{\frac{1}{q}}<\infty .
$$

(iii) $f \in C$ and

$$
\left|f\left(y_{00}\right)\right|+\left(\sum_{n=0}^{\infty} 2^{n s q}\left\|f-I_{2^{n}-1} f \mid L_{p}\right\|^{q}\right)^{\frac{1}{q}}<\infty .
$$

(iv) $f \in C$ and

$$
\left|f\left(y_{00}\right)\right|+\left(\sum_{n=0}^{\infty} 2^{n s q}\left\|I_{2^{n+1}-1} f-I_{2^{n}-1} f \mid L_{p}\right\|^{q}\right)^{\frac{1}{q}}<\infty .
$$

Proof. Step 1: First we show the finiteness of (24) - (26) if $f \in B_{p q}^{s}$. We employ similar techniques as in the proof of Theorem 3 . In view of the splitting in (18) and Proposition 2 for the finiteness of (24) it will be sufficient to deal with

$$
J=\left(\sum_{n=0}^{\infty}\left((1+n)^{s-\frac{1}{q}}\left\|I_{n}\left(f-\psi_{\left[\frac{n}{2}\right]} f\right) \mid L_{p}\right\|\right)^{q}\right)^{\frac{1}{q}} .
$$

For completeness note that

$$
\left|f\left(y_{00}\right)\right| \leq\left\|f|C\|\leq c\| f| B_{\infty}^{s-\frac{1}{p}}\right\| \leq c^{\prime}\left\|f \mid B_{p q}^{s}\right\|
$$

because of $s>\frac{1}{p}$ (cf. Remark 7 ). Let $d=\min (1, q)$. To estimate $J$ we apply

$$
\left\|I_{n}\left(f-\psi_{\left[\frac{n}{2}\right]} f\right)\left|L_{p}\left\|\leq c \sum_{\ell=0}^{\infty} 2^{\frac{\ell}{\rho}}\right\| f_{\ell+r-1}\right| L_{p}\right\|
$$

(cf. (20) and (21). Inserting this we obtain

$$
\begin{aligned}
J^{d} & \leq\left\{\sum_{r=0}^{\infty} \sum_{n=0}^{2^{r+1}-1} 2^{r s q} 2^{-r}\left(\sum_{\ell=0}^{\infty} 2^{\frac{\ell}{p}}\left\|f_{\ell+r-1} \mid L_{p}\right\|\right)\right\}^{\frac{d}{q}} . \\
& \leq c\left\|2^{r s d}\left(\sum_{\ell=0}^{\infty} 2^{\frac{\ell}{p}}\left\|f_{\ell+r-1} \mid L_{p}\right\|\right)^{d} \mid \ell_{q}\right\| \\
& \leq c \sum_{\ell=0}^{\infty}\left\|2^{r s d} 2^{\frac{\ell d}{p}}\right\| f_{\ell+r-1}\left|L_{p}\left\|^{d} \mid \ell_{q}\right\|\right. \\
& \leq c 2^{-\ell s d} 2^{\frac{\ell d}{p}}\left\|2^{(r+\ell) s d}\right\| f_{\ell+r-1}\left|L_{p}\left\|^{d} \mid \ell_{q}\right\|\right. \\
& \leq c^{\prime}\left\|f \mid B_{p q}^{s}\right\|^{d}
\end{aligned}
$$


because of $s>\frac{1}{p}$. By the above argument that proves finiteness of (24). The corresponding estimates in the cases (25) and (26) can be derived completely analoguous.

Step 2: If (24) or (25) is finite, then for $f \in C$ it follows $f \in B_{p q}^{s}$ by means of the characterization of Besov spaces given in Proposition 1.

Step 3: Let $f \in C$. Suppose that the expression in (26) is finite. We employ the same abbreviations as in proof of Theorem 3. Similar as there the following identity holds in $L_{p}$ :

$$
f_{\ell}(x)=\sum_{n=\ell-1}^{\infty} \sum_{k \in \mathbf{Z}}\left(I_{2^{n+1}-1} f-I_{2^{n}-1} f\right)^{\wedge}(k) \varphi_{\ell}(k) e^{i k x} \quad(\ell \geq 1)
$$

because of

$$
\left(I_{2^{n+1}-1} f-I_{2^{n}-1} f\right)^{\wedge}(k)=0 \quad \text { for }|k| \geq 2^{n+1}
$$

and $\lim _{n \rightarrow \infty} I_{n} f=f\left(\right.$ in $\left\|\cdot \mid L_{p}\right\| ;$ cf. [22: Subsection 10.7.14]). Hence,

$$
\left|f_{\ell}(x)\right| \leq \sum_{n=-1}^{\infty}\left|\left(I_{2^{n+\ell+1-1}} f-I_{2^{n+\ell-1}} f\right) \ell(x)\right|
$$

Again we put $d=\min (1, q)$. The Fourier multiplier theorem from [16: Theorem 3.3.4] gives us

$$
\sup _{\ell}\left\|\left(I_{2^{n+j+1}-1} f-I_{2^{n+j-1}} f\right) \ell\left|L_{p}\|\leq c\| I_{2^{n+j+1}-1} f-I_{2^{n+j}-1} f\right| L_{p}\right\|
$$

for some $c>0$ independent of $f$. All together this proves

$$
\begin{aligned}
\left(\sum_{\ell=1}^{\infty} 2^{\ell s q}\left\|f_{\ell} \mid L_{p}\right\|^{q}\right)^{\frac{d}{q}} & \leq c \sum_{n=-1}^{\infty}\left\|2^{\ell s d}\right\| I_{2^{n+\ell+1}-1} f-I_{2^{n+\ell-1}} f\left|L_{p}\left\|^{d} \mid \ell_{q}\right\|\right. \\
& \leq c \sum_{n=-1}^{\infty} 2^{-n s d}\left(\sum_{\ell=1}^{\infty} 2^{(\ell+n) s q}\left\|I_{2^{n+\ell+1}-1} f-I_{2^{n+\ell-1}} f \mid L_{p}\right\|^{q}\right)^{\frac{d}{q}} \\
& \leq c\left(\sum_{j=0}^{\infty} 2^{j s d}\left\|I_{2^{j+1}-1} f-I_{2^{j}-1} f \mid L_{p}\right\|^{q}\right)^{\frac{1}{q}}
\end{aligned}
$$

for some constant $c$ independent of $f$. It remains to estimate the term $\left\|f_{0} \mid L_{p}\right\|$. Again we employ

$$
\left.f=I_{0} f+\sum_{n=0}^{\infty}\left(I_{2^{n+1}-1} f-I_{2^{n}-1} f\right) \quad \text { (convergence in } L_{p}\right)
$$


(cf. [22: Subsection 10.7.14]) which implies

$$
\begin{aligned}
\left|f_{0}(x)\right| & =\left|f(0)+\hat{f}(1) e^{i x}+\hat{f}(-1) e^{-i x}\right| \\
& \leq 3 \int_{0}^{2 \pi}|f(x)| d x \\
& \leq \int_{0}^{2 \pi}\left|I_{0} f(x)\right| d x+\sum_{n=0}^{\infty} \int_{0}^{2 \pi}\left|\left(I_{2^{n+1}-1} f-I_{2^{n}-1} f\right)(x)\right| d x \\
& \leq(2 \pi)^{1-\frac{1}{p}}\left(\left\|I_{0} f\left|L_{p}\left\|+\sum_{n=0}^{\infty}\right\| I_{2^{n+1}-1} f-I_{2^{n}-1} f\right| L_{p}\right\|\right) \\
& \leq c\left(\left\|I_{0} f \mid L_{p}\right\|+\left(\sum_{n=0}^{\infty} 2^{n s q}\left\|I_{2^{n+1}-1} f-I_{2^{n}-1} f \mid L_{p}\right\|^{q}\right)\right)
\end{aligned}
$$

because of $s>0$. The proof is complete

Remark 10. We proved a little bit more than stated. In addition we showed that the expressions in (24) - (26) form equivalent quasi-norms in $B_{p q}^{s}$.

\section{Unconditional Schauder bases in $B_{p q}^{s}$}

There are several different approaches to construct unconditional Schauder bases in function spaces. After the break-through contributions of Ciesielski and collaborators, cf. e.g. [1] and [2], in case of Sobolev spaces those problems were extensively investigated. What concerns Besov spaces we refer to Ropela [13] and Oswald [9] for unconditional spline bases in $B_{p q}^{s}([0,1])$ (non-periodic case), Peetre [10], Triebel [21] and Sickel [18] for unconditional bases of analytic functions in $B_{p q}^{s}(\mathbb{R})$, Lizorkin [4], Orlovskij [7] and Schmeisser [15] for unconditional bases consisting of trigonometric polynomials for (generalized) periodic Besov spaces on the $n$-torus, or Meyer's book [5] for wavelet bases of several types.

Our approach is motivated by formula (26) and is similar to wavelet-techniques. For this we need to supplement our conditions on the matrices $Y$. In view of (26) it will be sufficient for us to consider submatrices. For a strictly increasing sequence $d=\left\{d_{n}\right\}_{n=0}^{\infty}$ of non-negative integers we say the pair $(Y, d)$ satisfies the regularity condition $\left(\mathcal{R}_{p, \sigma}\right)$ if the conditions of Definition 3 are satisfied at least for the rows with index $d_{n}$. Moreover, formula (26) motivates to concentrate on sequences with the mesh refinement property $\mathcal{M}$

$$
Y_{d_{n}} \subset Y_{d_{n+1}}, \quad n \geq 0 .
$$

To keep notations simple we put

$$
Y_{d_{n}}=W_{n}, \quad y_{d_{n} k}=w_{n k}, \quad W=\left\{W_{n}\right\}_{n}=\left\{w_{n k}\right\}_{n, k} .
$$


The corresponding interpolatory polynomials we denote by

$$
J_{n} f(x)=\sum_{k=0}^{2 d_{n}} f\left(w_{n k}\right) \ell_{d_{n} k}(x) \quad(n \geq 0) .
$$

If $(Y, d)$ satisfies the mesh refinement condition $(\mathcal{M})$, we have

$$
\begin{aligned}
\left(J_{n+1} f-J_{n} f\right)(x) & =\sum_{k=0}^{2 d_{n+1}}\left(f-J_{n} f\right)\left(w_{(n+1) k}\right) J_{d_{n+1}}(x) \\
& =\sum_{k \in W_{n+1} \backslash W_{n}}\left(f-J_{n} f\right)\left(w_{(n+1) k}\right) J_{d_{n+1}}(x) .
\end{aligned}
$$

Here $k \in W_{n+1} \backslash W_{n}$ is used as an abbreviation instead of $w_{(n+1) k} \in W_{n+1} \backslash W_{n}$. Because of $\left|W_{n}\right|=2 d_{n}+1$ we have $\left|W_{n+1}\right| W_{n} \mid=2\left(d_{n+1}-d_{n}\right)$. We introduce the functions

$$
\mathcal{L}_{n k}= \begin{cases}\ell_{d_{0} k} & \text { if } k=0,1, \ldots, 2 d_{0} \text { and } n=0 \\ \ell_{d_{n} k^{\prime}} & \text { if } k^{\prime} \in W_{n} \backslash W_{n-1} \text { and } n>0\end{cases}
$$

with an appropriate counting in the second line. So we associate to every nodal point $w_{n k}$ one fundamental function $\mathcal{L}_{n k}$.

4.1 Characterizations of Besov spaces by general sequences of interpolatory polynomials. Unfortunately, the dyadic sequence $\left\{2^{n}-1\right\}_{n=0}^{\infty}$ used in Theorem 4 does not satisfy the mesh refinement condition. For this reason we have to generalize our considerations from Subsection 3.3. First, observe the identity

$$
f=I_{d_{0}} f+\sum_{n=0}^{\infty}\left(I_{d_{n+1}} f-I_{d_{n}} f\right) \quad \text { (convergence in } L_{p} \text { ) }
$$

is valid for every. $f \in C$ and each sequence $\left\{d_{n}\right\}_{n=0}^{\infty}$ of strictly increasing non-negative integers. Second, we take into account the following equivalent characterization of periodic Besov spaces. Therefore, instead of the decomposition of unity defined in (14) we could take also systems $\left\{\varphi_{\ell}\right\}_{\ell=0}^{\infty}$ satisfying the following conditions:

(i) There exist two real numbers $\delta>1$ and $\alpha>\frac{1}{2}$ such that

$$
\operatorname{supp} \varphi_{0} \subset\left\{\xi:|\xi| \leq \delta^{\alpha}\right\} \quad \text { and } \quad \operatorname{supp} \varphi_{\ell} \subset\left\{\xi: \delta^{\ell-\alpha} \leq|\xi| \leq \delta^{\ell+\alpha}\right\} \quad(\ell \geq 1)
$$

(ii) For any $j \in \mathbb{N}_{0}$ there exist a positive constant $c_{j}$ such that

$$
\sup _{\ell \in N_{0}} \sup _{\xi \in \mathbb{R}} \delta^{j \ell}\left|\varphi_{\ell}^{(j)}(\xi)\right| \leq c_{j}<\infty
$$

(iii) There exist two positive constants $A$ and $B$ such that

$$
0<A \leq \sum_{\ell=0}^{\infty} \varphi_{\ell}(x) \leq B<\infty .
$$


Then

$$
B_{p q}^{s}=\left\{f \in D_{\pi}^{\prime}:\left\|f \mid B_{p q}^{s}\right\|^{*}=\left(\sum_{\ell=0}^{\infty} \delta^{\ell s q}\left\|\sum_{k \in \mathbf{Z}} \varphi_{\ell}(k) \hat{f}(k) e^{i k x} \mid L_{p}\right\|^{q}\right)^{\frac{1}{q}}<\infty\right\}
$$

$(q<\infty)$ and

$$
B_{p \infty}^{s}=\left\{f \in D_{\pi}^{\prime}:\left\|f\left|B_{p \infty}^{s}\left\|^{*}=\sup _{\ell \geq 0} \delta^{\ell s}\right\| \sum_{k \in \mathbf{Z}} \varphi_{\ell}(k) \hat{f}(k) e^{i k x}\right| L_{p}\right\|<\infty\right\} .
$$

Moreover, $\left\|f \mid B_{p q}^{s}\right\|^{*}$ yields an equivalent quasi-norm in $B_{p q}^{s}$ (cf. [3] or [20] for details in the non-periodic case).

Having these properties established then one could follow the proof of Theorem 4 step by step ending up with the following generalization.

Theorem 5. Let $1<p<\infty, 0<q \leq \infty$ and $s>\frac{1}{p}$. Let $Y$ be a matrix satisfying the regularity condition $\left(\mathcal{R}_{p, \sigma}\right)$. Moreover, assume that $\left\{d_{n}\right\}_{n=0}^{\infty}$ is a sequence of strictly increasing non-negative integers such that there exist $\delta>1$ and $\alpha, \beta$ with $0<\alpha<\beta$ such that

$$
0<\alpha \leq \frac{d_{n}}{\delta^{n}} \leq \beta<\infty \quad(n \geq 1)
$$

Then $f$ belongs to $B_{p q}^{s}$ if and only if $f \in C$ and

$$
\delta^{n s}\left\|I_{d_{n+1}} f-I_{d_{n}} f \mid L_{p}\right\| \in \ell_{q}
$$

Moreover, the expression

$$
\left\|I_{d_{0}} f \mid L_{p}\right\|+\left(\sum_{n=0}^{\infty} \delta^{n s q}\left\|I_{d_{n+1}} f-I_{d_{n}} f \mid L_{p}\right\|^{q}\right)^{\frac{1}{q}}
$$

(modification if $q=\infty$ ) yields an equivalent quasi-norm in $B_{p q}^{s}$.

4.2 Unconditional Schauder bases in $B_{p, q}^{*}$. Based on Theorem 5 and the observation made in (28) it becomes now easy to construct Schauder bases.

Theorem 6. Let $1<p<\infty, 0<q<\infty$ and $s>\frac{1}{p}$. Let $d=\left\{\left\{d_{n}\right\}_{n=0}^{\infty}\right.$ be a strictly increasing sequence of non-negative integers which fulfils (31) for some $\delta>1$ and $0<\alpha<\beta<\infty$. Further we assume that the pair $(Y, d)$ satisfies the regularity condition $\left(\mathcal{R}_{p, \sigma}\right)$ and the mesh refinement condition $(\mathcal{M})$. Let $\mathcal{L}_{n k}$ be the functions introduced in (29). Then the set

$$
\mathcal{L}(Y, d)=\left\{\mathcal{L}_{0 k}: k=0, \ldots, 2 d_{0}\right\} \cup\left\{\mathcal{L}_{n k}: \dot{k}=1,2, \ldots, 2\left(d_{n}-d_{n-1}\right), n \geq 1\right\}
$$

yields an unconditional Schauder bases for the periodic Besov spaces $B_{p q}^{s}$. 
Proof. We know that every $f \in B_{p q}^{s}$ can be represented as

$$
\begin{aligned}
f & =\dot{I}_{d_{0}} f+\sum_{n=0}^{\infty}\left(I_{d_{n+1}} f-I_{d_{n}} f\right) \\
& =\sum_{k=0}^{2 d_{0}} \alpha_{0 k} \mathcal{L}_{0 k}+\sum_{n=1}^{\infty} \sum_{k=1}^{2\left(d_{n}-d_{n-1}\right)} \alpha_{n k} \mathcal{L}_{n k} \quad \text { (convergence in } L_{p} \text { ) }
\end{aligned}
$$

for suitable chosen $\alpha_{n k} \in \mathbb{C}$ (cf. (28)). The representation converges in $\left\|\cdot \mid B_{p q}^{s}\right\|$, that is implied by. Theorem 5 and $q<\infty$. The stability of the representation in (33) (with respect to the $\alpha_{n k}$ ) follows from Theorems 1 and 2 ; in fact, we have

$$
\begin{aligned}
& \frac{1}{C_{p}}\left(\frac{1}{2 d_{n+1}+1} \sum_{k \in W_{n+1} \backslash W_{n}}\left|\left(f-J_{n} f\right)\left(w_{(n+1) k}\right)\right|^{p}\right)^{\frac{1}{p}} \\
& \leq\left\|\sum_{k \in W_{n+1} \backslash W_{n}} \alpha_{(n+1) k} \mathcal{L}_{(n+1) k} \mid L_{p}\right\| \\
& \quad=\left\|I_{d_{n+1}} f-I_{d_{n}} f \mid L_{p}\right\| \\
& \leq D_{p}\left(\frac{1}{2 d_{n+1}+1} \sum_{k \in W_{n+1} \backslash W_{n}}\left|\left(f-J_{n} f\right)\left(w_{(n+1) k}\right)\right|^{p}\right)^{\frac{1}{p}} .
\end{aligned}
$$

Because of

$$
\alpha_{(n+1) k}=\left(f-J_{n} f\right)\left(w_{(n+1) k}\right)
$$

unconditionality is implied by Theorem 5 . From that uniqueness of the representation in (33) becomes obvious (cf. also the following remark, in particular (36))

Remark 11. Again we have proved more than stated. If $f \in C$ is given by

$$
f=\sum_{k=0}^{2 d_{0}} \alpha_{0 k} \mathcal{L}_{0 k}+\sum_{n=1}^{\infty} \sum_{k=1}^{2\left(d_{n}-d_{n-1}\right)} \alpha_{n k} \mathcal{L}_{n k}
$$

then

$$
\left(\frac{1}{2 d_{0}+1} \sum_{k=0}^{2 d_{0}}\left|\alpha_{0 k}\right|^{p}\right)^{\frac{1}{p}}+\left\{\sum_{n=1}^{\infty} \delta^{n s q}\left(\frac{1}{2 d_{n}+1} \sum_{k=1}^{2\left(d_{n}-d_{n-1}\right)}\left|\alpha_{n k}\right|^{p}\right)^{\frac{q}{p}}\right\}^{\frac{1}{p}}
$$

yields an equivalent quasi-norm on $B_{p q}^{s}$. Here $\delta$ is the number for which $d$ satisfies (31). Taking into account

$$
\left.\begin{array}{c}
\alpha_{0 k}=f\left(w_{0 k}\right) \quad\left(k=0,1, \ldots, 2 d_{0}\right) . \\
\alpha_{(n+1) k}=f\left(w_{(n+1) k}\right)-\sum_{\ell=0}^{2 n} f\left(w_{n \ell}\right) \prod_{j \in\left\{0,1, \ldots, 2 d_{n}\right\} \backslash\{\}} \frac{\sin \left(\frac{w_{(n+1) k}-w_{n j}}{2}\right)}{\sin \left(\frac{w_{n \ell}-w_{n j}}{2}\right)} \\
\left(k=0,1, \ldots, 2\left(d_{n+1}-d_{n}\right), n \geq 0\right)
\end{array}\right\}
$$


(cf. (35)), we have obtained an explict formula for $\left\|f \mid B_{p q}^{s}\right\|$ (in the sense of an equivalent norm) using only weighted means of values of $f$ at the points $\left\{w_{n k}\right\}_{n, k}$.

\section{Examples}

5.1 Uniformly distributed knots. We have to show existence of sequences $d=$ $\left\{d_{n}\right\}_{n}$ and of matrices $Y$ satisfying the conditions of Theorem 6 . A natural family of admissible matrices and corresponding sequences $\left\{d_{n}\right\}_{n=0}^{\infty}$ consists in the following. Having in one level a uniform distribution of knots, then we subdivide the corresponding interval to the right of each knot into $\ell$ subinterval of equal length for some $\ell \geq 2$. That means we require

$$
2 d_{n+1}+1=\ell\left(2 d_{n}+1\right) \quad(n \geq 0)
$$

for some $\ell \in \mathbb{N} \backslash\{1\}$. Then the number of knots in level $n+1$ is given by a constant multiple of the number of knots of the preceeding level. Since the numbers $d_{n}$ have to be integers it follows from

$$
d_{n+1}=\ell d_{n}+\frac{\ell-1}{2}
$$

that $\ell$ has to be an odd number. For simplicity we require in addition $d_{0}=0$ and $d_{1}=1$. We end up with

$$
d_{n+1}=\frac{3}{2} \ell^{n}-\frac{1}{2} \quad(n \geq 0) .
$$

The knots are required to be equally distributed, hence given by

$$
\left.\begin{array}{rl}
w_{00} & =0 \\
w_{n k} & =\frac{2 \pi}{3} \ell^{-n+1} k \quad\left(k=0,1, \ldots, 3 \ell^{n-1} .\right.
\end{array}\right\}
$$

The corresponding matrix $W^{\ell}$ satisfies the conditions $\left(\mathcal{R}_{p, \sigma}\right)$ as well as $(\mathcal{M})$ if $\ell$ is an odd natural number larger than 1.

5.2 Non-uniformly distributed knots. More general bases may be obtained by destroying the regular structure of these sets of knots in a certain controlled way. For instance, we may take

$$
\widetilde{w}_{n k}=w_{n k}+\frac{2 \pi}{3} \ell^{-n-\gamma_{n k}} \varepsilon_{n k} \quad\left(k \in W_{n} \backslash W_{n-1}, n \geq 1\right) .
$$

There $\varepsilon_{n k} \in\{0,1\}$ can be chosen arbitrarily. The only requirement with respect to $\gamma_{n k}$ consists in $\gamma_{n k} \in \mathbb{N}$. To obtain the regularity of a corresponding matrix we need to have

$$
\frac{2 \pi}{3 \ell}<\sigma<\sigma_{p}
$$

Let $r \in \mathbb{N}$. Having this condition at hand we get

$$
\begin{aligned}
\tilde{w}_{n k} & =\frac{2 \pi}{3} \ell^{-n+1} k+\varepsilon_{n k} \frac{2 \pi}{3} \ell^{-n-\gamma_{n k}} \\
& =\frac{2 \pi}{3} \ell^{-n-r+1}\left(k \ell^{r}\right)+\varepsilon_{n k} \frac{2 \pi}{3} \ell^{-n-\gamma_{n k}} \\
& =\tilde{w}_{(n+r)\left(k \ell^{r}\right)} .
\end{aligned}
$$


As long as $r-1<\gamma_{n k}(40)$ implies

$$
\frac{2 \pi(k-\sigma)}{2 d_{n+r}+1} \leq \widetilde{w}_{(n+r) k} \leq \frac{2 \pi(k+\sigma)}{2 d_{n+r}+1} .
$$

In case $r-1=\gamma_{n k}$ we have

$$
\widetilde{w}_{(n+r) k}=w_{(n+r)(k \ell+1)} .
$$

If such a situation occurs, we include also $w_{(n+r)(k / r)}$ in our knot sequence of level $n+r$. By an appropriate counting this modified sequence, now denoted by $v_{(n+r) k}$, also satisfies

$$
\frac{2 \pi(k-\sigma)}{2 d_{n+r}+1} \leq v_{(n+r) k} \leq \frac{2 \pi(k+\sigma)}{2 d_{n+r}+1} .
$$

Hence, the above construction leads to a family of matrices $V^{\ell}=\left\{v_{n k}\right\}_{n, k}$ satisfying the conditions $\left(\mathcal{R}_{p, \sigma}\right)$ and $(\mathcal{M})$ and being different from the "regular" case $W^{\ell}$.

Remark 12. The above examples show that there exist matrices $Y$ and sequences $d$ satisfying the conditions of Theorem 6 . Hoewever, of interest would be a more general stability result corresponding to the regular cases $W^{\ell}$ (cf. (38)).

Acknowledgement The authors would like to thank the referee for pointing out to us Theorem 1.

\section{References}

[1] Ciesielski, Z. and J. Domsta: Construction of an orthonormal basis in $C^{m}\left(I^{d}\right)$ and $W_{p}^{m}\left(I^{d}\right)$. Studia Math. 41 (1972), $211-224$.

[2] Ciesielski, Z. and T. Figiel: Construction of Schauder bases in function spaces on smooth compact manifolds. In: Approximation and Function Spaces, Gdansk 1979 (ed.: Z. Ciesielski). Amsterdam: North Holland 1981, pp. $217-232$.

[3] Kalyabin, G. A. and P. L. Lizorkin: Spaces of functions of generalized smoothness. Math. Nachr. 133 (1987), 7 - 32.

[4] Lizorkin, P. I.: On bases and multipliers for the spaces $B_{p, \Theta}^{r}$. Trudy Math. Inst. Steklov 143 (1977), $88-104$.

[5] Meyer, Y.: Wavelets and Operators. Cambridge: Univ. Press 1992.

[6] Nikol'skij, S. M.: Approximation of Functions of Several Variables and Imbedding Theorems. Berlin: Springer-Verlag 1975.

[7] Orlovskij, D. G.: On multipliers in the spaces $B_{p, \Theta}^{r}$. Anal. Math. 5 (1979), $207-218$.

[8] Oskolkov, K. I.: Inequalities of "large sieve" type and applications to trigonometric approximation. Anal. Math. 12 (1986), $143-166$.

[9] Oswald, P.: On inequalities for spline approximation and spline systems in the spaces $L_{p}(0<p<1)$. In: Approximation and Function spaces (ed.: Z. Ciesielski). Amsterdam: North Holland 1981, pp. 531 - 552.

[10] Peetre, J.: New Thoughts on Besov Spaces (Duke Univ. Math. Series). Durham: Duke Univ. 1976. 
[11] Pichorides, S. K.: On the best values of the constants in the theorems of M. Riesz, Zygmund and Kolmogorov. Studia Math. 44 (1972), 165 - 179.

[12] Prestin, J.: Trigonometric interpolation of functions of bounded variation. In: Constructive Theory of Functions. Proc. Conf. Varna 1984 (ed.: B. Sendov, P. Petrushev, R. Maleev and S. Tashev). Sofia: Publ. House Bulg. Acad. Sci. 1984, pp. $699-703$.

[13] Ropela, S.: Spline bases in Besov spaces. Bull. Acad. Polon. Sci., Ser. Sci. Math. Astronom. Phys. 24 (1976), 476 - 470.

[14] Runovski, K. V.: On families of linear polynomial operators in $L_{\mathrm{p}}$-spaces, $0<p<1$. Russian Acad. Sci. Sb. Math. 78 (1994), 165 - 173.

[15] Schmeisser, H.-J.: An unconditional basis in periodic spaces with dominating mixed smoothness properties. Anal. Math. 13 (1987), 153 - 168.

[16] Schmeisser, H.-J. and H. Triebel: Topics in Fourier Analysis and Function Spaces. Chichester: Wiley 1987.

(17) Sickel, W.: Some remarks on trigonometric interpolation on the n-torus. Z. Anal. Anw. 10 (1991), $551-562$.

[18] Sickel, W.: Characterization of Besov-Triebel-Lizorkin spaces via approximation by Whittakers cardinal series and related unconditional Schauder bases. Constr. Approx. 8(1992), $257-274$.

[19] Stechkin, S. B.: Generalization of some inequalities of S. N. Bernstein. Dokl. Akad. Nauk SSSR 60 (1948), 1511 - 1514.

[20] Triebel, H.: Fourier Analysis and Function Spaces (Teubner-Texte zur Mathematik: Vol. 7). Leipzig: B. G. Teubner Verlagsges. 1977.

[21] Triebel, H.: Theory of Function Spaces. Basel: Birhhäuser Verlag 1983.

[22] Zygmund, A.: Trigonometric Series. Volumes I and II. Cambridge: Univ. Press 1977. 